\section{Dezentralisierung der Energieversorgung} Herausforderungen an die Systemanalyse und -steuerung

von Dominik Möst, TU Dresden, sowie Patrick Jochem und Wolf Fichtner, IIP/KIT

Der Anteil der Elektrizitätsbereitstellung aus dezentralen Anlagen, im Wesentlichen auf Basis erneuerbarer Energien und der KraftWärme-Kopplung, wird in den kommenden Jahren erheblich ansteigen. Bis zum Jahr 2050 könnte der Beitrag der erneuerbaren Energien an der Elektrizitätserzeugung bis zu 100 Prozent betragen. Die damit verbundene Dezentralisierung der Energieversorgung stellt neue Herausforderungen u. a. an den Betrieb des Systems. An dieser Stelle setzt die Energiesystemanalyse an, die die Auswirkungen der zu erwartenden Entwicklungen auf das Energiesystem abschätzt. In diesem Beitrag wird im ersten Abschnitt auf die zu erwartenden Entwicklungen im Energiemarkt und die damit verbundenen Herausforderungen eingegangen, um dann im zweiten Abschnitt die Auswirkungen auf die Energiesystemanalyse abzuleiten.

\section{Einleitung}

Eine zuverlässige und wirtschaftliche Energieversorgung ist von großer Bedeutung für die Entwicklung von heutigen Industriegesellschaften. Politische Entscheidungsträger haben insofern großes Interesse, die Entwicklungen auf Energiemärkten zu kontrollieren, und üben durch Auferlegung rechtlicher Rahmenbedingungen politischen Einfluss auf die Branche aus. ${ }^{1}$ Während dieser politische Einfluss in der Vergangenheit in erster Linie national war, wird dieser in Europa heutzutage zunehmend durch die Organe der Europäischen Union ausgeübt. Durch diesen Einfluss haben sich die energiewirtschaftlichen Rahmenbedingungen in den vergangenen Jahren stark verändert. Insbesondere die Liberalisierung der europäischen Energiemärkte hat hierbei eine zentrale Rolle gespielt. Aber auch für die kommenden Dekaden werden weitere tiefgreifende Veränderungen erwartet. Dies wird u. a. durch die internationalen Verpflichtungen zur Treibhausgasemissionsreduktion begründet. Die Europäische Union verpflichtete sich, Treibhausgasemissionen bis 2020 um mindestens $20 \%$, ggf. sogar um 30 \% (sofern vergleichbare Reduktionen in anderen Ländern angestrebt werden) gegenüber dem Basisjahr 1990 zu reduzieren. Ferner soll der Endenergiebedarf bis $2020 \mathrm{zu}$ mindestens $20 \%$ aus Erneuerbaren Energien gedeckt werden. Die Bundesregierung hat sich darüber hinaus das Ziel gesetzt, bis 2020 den Anteil Erneuerbarer Energien an der Stromversorgung auf mindestens $35 \%$ zu erhöhen und den Anteil der Strombereitstellung aus Kraft-Wärme-Kopplungsanlagen auf etwa $25 \%$ zu verdoppeln. Anhand dieser Zahlen ist ersichtlich, dass die Elektrizitätsbereitstellung aus dezentralen Anlagen auf Basis erneuerbarer Energien und der KraftWärme-Kopplung in den kommenden Jahren bedeutende Anteile erreichen wird.

Neben diesen Zielen im Energiesektor hat sich die Europäische Union auch ehrgeizige Ziele im Verkehrssektor gesetzt, um in diesem Sektor dem Trend des $\mathrm{CO}_{2}$-Emissionsanstiegs in den letzten Dekaden entgegenzuwirken. Eine Maßnahme hierbei ist die Förderung der Elektromobilität. Diese erlaubt zwar keine emissionslose Mobilität, ermöglicht aber eine erhebliche Emissionssenkung im Personenstraßenverkehr. Lokal können lediglich stark gesenkte Lärmemissionen und ggf. wenige Partikel gemessen werden. Die Gesamtemissionen sind beim elektrischen Fahren stark von der Elektrizitätserzeugung abhängig und somit faktisch in den Energieerzeugungssektor verschoben. Elektrisches Fahren führt demnach zu einer größeren Nachfrage nach Elektrizität und stellt somit eine weitere Herausforderung an das Energiesystem. Aber es könnte in Zukunft in einem stark dezentralisierten Energiesystem auch einen Beitrag zu einem zuverlässigeren System leisten - falls die Fahrzeuge vorübergehend als Energiespeicher zur Verfügung stünden.

Diese zu erwartende Dezentralisierung der Energieversorgung macht eine verlässliche Elektrizitätsbereitstellung immer komplexer und damit die Energiesystemanalyse, die die Auswirkungen der zu erwartenden Entwicklungen auf das Energiesystem abschätzt, unerlässlich. Die Energiesystemanalyse soll helfen, Entscheidungen in 
Energiepolitik und Energieforschung im Hinblick auf Technologien und Infrastrukturen für Energiebereitstellung und Energieumwandlung wissensbasiert und systematisch zu unterstützen. In diesem Beitrag wird deshalb im ersten Abschnitt auf die zukünftigen Entwicklungen und die Herausforderungen im Energiemarkt eingegangen, um dann im zweiten Abschnitt die Auswirkungen auf die Energiesystemanalyse abzuleiten. ${ }^{2}$

\section{Herausforderungen und Unsicherheiten in der Energieversorgung}

\subsection{Dezentralisierung der Energieversorgung}

\subsubsection{Entwicklung von erneuerbaren Energien}

Sowohl auf der EU-Ebene als auch auf nationaler Ebene sind konkrete Ziele und Maßnahmen vereinbart worden, um einen weiteren Ausbau erneuerbarer Energien voranzutreiben. In der Direktive 2009/28/EC werden Ausbauziele für erneuerbare Energien bezogen auf den Gesamtenergiebedarf (nicht nur Elektrizität) für das Jahr 2020 definiert. Deutschland soll hiernach seinen Anteil der Energie aus erneuerbaren Quellen am Bruttoendenergieverbrauch von 5,8\% im Jahr 2005 auf $18 \%$ im Jahr 2020 erhöhen. Auf nationaler Ebene ist 2010 von der Bundesregierung das Energiekonzept vorgelegt worden, in dem die europäischen Rahmenbeschlüsse bezüglich des Ausbaus Erneuerbarer Energien, der Energieeffizienz und des Klimaschutzes aufgegriffen bzw. teilweise sogar übertroffen werden. Danach ist eine Erhöhung des Anteils auf $30 \%$ bis $2030,45 \%$ bis 2040 und $60 \%$ bis $2050 \mathrm{im}$ Energiekonzept des Jahres 2010 der Bundesregierung angestrebt. Dabei ist der Wärmesektor von entscheidender Bedeutung, da mehr als die Hälfte der gesamten Endenergie, die in Deutschland genutzt wird, für die Wärmebereitstellung benötigt wird (BMWi 2010). Der Elektrizitätssektor soll aufgrund der verfügbaren Potenziale überproportional zum EU-Ziel für erneuerbare Energien beitragen. Im Jahr 2020 soll der Anteil der Stromerzeugung aus erneuerbaren Energien am Bruttostromverbrauch $35 \%$ ausmachen. Anschließend soll der Anteil auf 50\% bis 2030 und $80 \%$ in 2050 gesteigert werden. Hieraus ist ersichtlich, dass verschiedene Technologien auf Basis erneuerbarer Energien zur Erreichung der Ziele herangezogen werden müssen. Zudem entstehen durch den fluktuierenden Charakter der Wind- und PV-Einspeisung besondere Herausforderungen für das Energiesystem. Die zunehmenden Anteile aus fluktuierenden Quellen werden das künftige Energiesystem entscheidend determinieren. Dies betrifft sowohl den Speicherbedarf von elektrischer Energie und die Verdrängung von Grundlastkraftwerken als auch den Ausbau der Übertragungs- und ggf. auch der Verteilungsnetze.

Für den Elektrizitätssektor wird für Deutschland im Jahr 2020 mit einem Beitrag zur Stromerzeugung von Offshore-Windenergieanlagen von ca. 34 TWh gerechnet (BMU 2009). Bei einer angenommenen Volllaststundenzahl von $3.500 \mathrm{~h}$ für Offshore-Windenergieanlagen entspräche dies einer installierten Leistung von ca. 10 GW. Im Gegensatz zu anderen Studien wird von einem langsameren Offshore-Windenergieausbau ausgegangen, der sich heute bereits so abzeichnet. Der Beitrag von OnshoreWindenergieanlagen liegt bei ca. $54 \mathrm{TWh}$ im Jahr 2020 (BMU 2009), woraus sich eine installierte Leistung von $29 \mathrm{GW}$ bei einer angenommen Volllaststundenzahl von $1.900 \mathrm{~h}$ ergibt. Bei der Photovoltaik, welche insbesondere im Gegensatz zur Offshore-Windenergie, in der Regel eine dezentrale Technologie ist, wird von einer Stromerzeugung von ca. 15,5 TWh bis zum Jahr 2020 ausgegangen (BMU 2009). Die installierte Kapazität entspräche unter der Annahme von 1.100 Volllaststunden ca. $14 \mathrm{GW}$, wobei diese Zahl als zu niedrig einzustufen ist, da diese Kapazität voraussichtlich bereits im Jahr 2011 erreicht werden wird. $^{3}$ Auch andere Studien sehen ähnliche Ausbauraten bei den erneuerbaren Energien vor, so dass zukünftig mit einem hohen Anteil fluktuierender Stromeinspeisung aus erneuerbaren Energien zu rechnen ist. Eine Zählstatistik der 1h-Variationen der Windenergieeinspeisung kann die Größenordnung der Fluktuationen verdeutlichen. Es überwiegen zwar diejenigen Ereignisse, in denen die Variationen der Einspeisung zwischen einer Stunde und der nächsten im Bereich von null bis zehn Prozent der installierten Leistung liegen, den- 
noch treten bei Offshore-Windenergieanlagen auch Schwankungen von über 20 Prozent der installierten Leistung auf (Genoese et al. 2009). Bei einer installierten Windenergieleistung von ca. $39 \mathrm{GW}$ in 2020 könnten die Fluktuationen dann bereits schon ca. $8 \mathrm{GW}$ pro Stunde betragen und wären somit von großer Bedeutung für einen zuverlässigen Betrieb des Energiesystems. Hierzu müssen geeignete Maßnahmen umgesetzt und entsprechende Rahmenbedingungen geschaffen werden, um derartige Situationen im Netzbetrieb sicher zu beherrschen. Bessere Vorhersagen der fluktuierenden Einspeisung, eine kurzfristige flexible Anpassung der Erzeugungseinheiten (auch der planbaren erneuerbaren Kapazitäten) und die Steuerung der Nachfrageseite sind deshalb zentrale Anforderungen in einem zukünftigen Energiesystem und damit auch für die Energiesystemanalyse. Darüber hinaus besteht die Hoffnung, dass durch die großflächige Vernetzung der erneuerbaren Energien (bspw. durch eine Hochspannungsgleichstromübertragung) extreme Fluktuationen durch natürliche, weiträumige Ausgleichseffekte verringert werden können. Auch hier kann die Energiesystemanalyse helfen, mit entsprechend fortentwickelten Modellen die Auswirkungen einer großflächigen Vernetzung auf das Energiesystem abzuschätzen.

\subsubsection{Der europäische Energiemarkt und Netzengpässe im Übertragungsnetz}

Ziel der Liberalisierung war es, die staatlichen Monopole in der Energieversorgung durch wettbewerbliche, offene, integrierte und effiziente Energiemärkte zu ersetzen. Vor allem sollte ein freier und wettbewerbsorientierter europäischer Binnenmarkt geschaffen werden. Mit der Liberalisierung und der Einführung des Wettbewerbs stieg der grenzüberschreitende Stromaustausch kontinuierlich an und Übertragungskapazitäten zwischen verschiedenen Ländern werden nun häufig an ihrem Kapazitätslimit betrieben (z. B. Schweiz nach Italien, Frankreich nach Italien, s. Haubrich et al. 2002). Auch auf nationaler Ebene kommt es inzwischen zu Netzengpässen, zukünftig wird sich diese Situation voraussichtlich noch erheblich verschlimmern, da - wie im vorherigen Abschnitt beschrieben - die Kapazität von erneuerbaren (fluktuierenden) Energien aufgrund der gezielten Förderung weiterhin stark ansteigen wird. Die geografische Verteilung der erneuerbaren Anlagen ist aufgrund der regional unterschiedlichen Verfügbarkeit der erneuerbaren Quellen sehr heterogen. Da die Einspeisung aus erneuerbaren Energien in das Übertragungsnetz häufig weit von den Verbrauchern (v. a. bedingt durch den wachsenden Anteil von Windenergie) entfernt ist, gibt es einen erheblichen Einfluss auf den Lastfluss in den nationalen Übertragungsnetzen. Aufgrund des Trends zu einem zunehmenden Nord-SüdGefälle der installierten Windenergieanlagen sowie der Tatsache, dass das Transportnetz ursprünglich nicht für hohe Transite ausgelegt worden ist, werden in Deutschland zukünftig vermehrt Engpässe vornehmlich in Nord-SüdRichtung auftreten. ${ }^{4}$ Um diesen Engpässen im Übertragungsnetz entgegenzuwirken, können drei Strategien verfolgt werden. Zum einen kann versucht werden die Last geografisch zu verlagern. Dies ist allerdings mit einer Umsiedlung von Industriestandorten oder der Bevölkerung nur schwierig zu realisieren. Jedoch könnten immerhin langfristig lokale Preisnachlässe die Standortwahl energieintensiver Industrien beeinflussen. Kurzfristig könnte eine zeitliche Nachfragesteuerung der Elektrizitätskunden zeitlich begrenzter Engpässe entgegenwirken. Zweitens können auch auf der Angebotsseite Anreize gegeben werden, Erzeugungskapazitäten langfristig in der Engpassregion anzusiedeln. Als dritte Option kann der Netzausbau verstärkt werden, um die Übertragungskapazität massiv zu erhöhen. Marktorientierte Preisgestaltungskonzepte für die Übertragungsnetze können hier ggf. die richtigen Anreize setzen und werden an Bedeutung gewinnen (Europäische Kommission 2004a). Allerdings stellt sich die Frage, welche Auswirkungen sich durch solche Marktgestaltungen auf das Energiesystem ergeben. In diesem Zusammenhang kann die Energiesystemanalyse helfen, die Auswirkungen von den unterschiedlichen Entwicklungen systematisch und wissensbasiert zu untersuchen, um damit Entscheidungsträgern eine quantitative Unterstützung zu geben. 


\subsubsection{Energieeffizienz, die Integration von Endkunden und Elektromobilität}

Neben den erneuerbaren Energien und den Netzen gibt es insbesondere noch drei weitere Themen, die den Elektrizitätsmarkt in den nächsten Dekaden verändern werden. Neben dem durch die Medien stark verbreiteten Thema der Elektromobilität ist im Hinblick einer wirtschaftlichen Klimagasreduktion insbesondere die Energieeffizienz ein wichtiger Bestandteil des künftigen Energiesystems. Hierzu kann eine stärkere Anbindung von Endverbrauchern an die Elektrizitätsmärkte einen entscheidenden Beitrag leisten. Im April 2006 hat die Europäische Kommission die Richtlinie 2006/32/EG (Europäische Kommission 2006) über Endenergieeffizienz und Energiedienstleistungen veröffentlicht, in der „die Effizienz der Endenergienutzung in den Mitgliedsstaaten ... [gesteigert werden soll und zwar durch die] Festlegung der erforderlichen Richtziele sowie der erforderlichen Mechanismen, Anreize und institutionellen, finanziellen und rechtlichen Rahmenbedingungen zur Beseitigung vorhandener Markthindernisse und -mängel, die der effizienten Endenergienutzung entgegenstehen [sowie der] Schaffung der Voraussetzungen für die Entwicklung und Förderung eines Marktes für Energiedienstleistungen (...)“. Im neunten Jahr nach Verabschiedung der Richtlinie ist der Energieeinsparrichtwert von 9 Prozent zu erreichen. Energieaudits, intelligente Verbrauchmesssysteme, informative Abrechnungen und energieeffiziente Tarife sind ausdrücklich als Maßnahmen zur Erreichung dieses Ziels genannt. In Kombination mit zeitlich variierenden Strompreisen sollen diese Maßnahmen die Integration der gegenwärtig eher passiven Endkunden in den Strommarkt integrieren und die damit verbundene bisher unelastische Nachfrage flexibilisieren. D. h. die Nachfrage soll in gewissen Bandbreiten durch zeitabhängige Preissignale und der Möglichkeit des Endkunden darauf $\mathrm{zu}$ reagieren, gezielt gesteuert werden. Hierbei spielen insbesondere die im Haushalt steuerbaren Technologien eine wichtige Rolle. Insbesondere in der Wärmebereitstellung werden große Potenziale zur Energieeffizienz und zur Lastverlagerung gesehen, bspw. durch gezieltes Schalten von Wärmepumpen, Nachtspeicherheizungen und Kraft-Wärme-Kopplungsanlagen (KWK). Insbe- sondere KWK-Anlagen sollen zukünftig laut dem Integrierten Energie- und Klimaprogramm der Bundesregierung weiter an Bedeutung gewinnen, da der Anteil der KWK-Stromerzeugung bis zum Jahr 2020 auf 25 Prozent verdoppelt werden soll (BMU 2007).

Als wesentliche Maßnahme zur Erreichung des Ziels wurde das Kraft-Wärme-Kopplungsgesetz novelliert, welches Netzbetreiber verpflichtet, KWK-Anlagen anzuschließen, den erzeugten Strom abzunehmen und zu vergüten. Bisher wird KWK-Strom größtenteils in Anlagen ab einer Leistung größer als ein MW erzeugt, insbesondere in der Industrie oder in der Wärmeversorgung mit Wärmenetzen (Roon, Steck 2009). Allerdings gewinnen Anlagen mit kleinerer Leistung, sog. Mikro-KWK-Anlagen, zunehmend für die Hausenergieversorgung an Bedeutung und tragen damit ebenso zu einer weiteren Dezentralisierung der Energieversorgung bei. Die Obergrenze für die elektrische Leistung der Mikro-KWK-Anlagen ist unterschiedlich definiert und wird vom Europäischen Parlament bei $50 \mathrm{~kW}_{\text {el }}$ gezogen (Europäische Kommission 2004b). Ein Grund für den gegenwärtig noch geringen Anteil von Mikro-KWK-Anlagen liegt in den hohen Kapitalkosten im Vergleich zu konventionellen Heizkesseln. Das von Lichtblick ${ }^{5}$ angekündigte Konzept versucht diese Hürde durch eine niedrigere Investition bei gleichzeitiger Gewährung eines Wärmepreises unterhalb der Wärmeerzeugungskosten mit einem Erdgas-Brennwertkessel zu überwinden. Dafür liegen das Fahrplanmanagement und die Vermarktung des erzeugten Stroms in der Verantwortung von Lichtblick. Lichtblick schätzt die Installation auf 100.000 Anlagen mit einer elektrischen Leistung von $19 \mathrm{~kW}_{\mathrm{el}}$ Damit würde die Gesamtleistung der Anlagen ca. 1,9 GW betragen und in der Größenordnung von ein bis zwei Großkraftwerken liegen. Die Umsetzung allein dieses Konzepts könnte somit wesentlich zu einer gesteuerten dezentralen Einspeisung beitragen. Allerdings ist anzumerken, dass die Einspeisung elektrischer Energie nur in einer gewissen Bandbreite möglich ist, da Restriktionen durch die Wärmenutzung zu berücksichtigen sind.

Während Mikro-KWK-Anlagen insbesondere an windarmen Wintertagen bereits eine geeignete Ergänzung zu regenerativer Energie- 
erzeugung sind und somit zu einer Entlastung der bisherigen Kraftwerksstruktur führen, ist der Einfluss der Elektromobilität auf die Energiewirtschaft erst bei höheren Penetrationen relevant für das Energiesystem. Eine geringe Anzahl an Fahrzeugen auf dem Markt hat nur sehr geringe Einflüsse - wohingegen hohe Penetrationsraten von (theoretisch) bis zu 40 Mio. Pkw in Deutschland die Elektrizitätsnachfrage um ca. 20 Prozent steigern würden. Problematisch wäre hierbei insbesondere die Auswirkung auf die „Tagesganglinie": Gerade in den Abendstunden, in denen bereits heute die Netze am stärksten ausgelastet sind, würden die meisten Fahrzeuge geladen und somit zumindest lokal Netze überlastet werden.

Um dies zu verhindern, können die Fahrzeuge zeitlich versetzt geladen werden (kontrolliert unidirektionales Laden) und damit neben dem Entlasten der Netze in den Abendstunden $\mathrm{zu}$ einem wirtschaftlicheren Betrieb nicht voll ausgelasteter Grundlastkraftwerke beitragen. Ein weiterer Schritt wäre beim Ladevorgang des Fahrzeugs auch ein Rückspeisen zuzulassen (kontrolliert bidirektionales Laden, oder auch vehicle-to-grid, kurz V2G). Diese Alternative ist insbesondere bei einem hohen Windenergieanteil am Markt eine geeignete Variante, um mit einer großen Anzahl an Fahrzeugen unvorhergesehene Windeinspeiseschwankungen abzufedern. Die Rentabilität dieser Speichertechnologie ist jedoch noch weitgehend unklar. Bisher sind Pumpspeicherkraftwerke (zumindest in entsprechend geeigneten Regionen) die wesentlich günstigere Alternative.

Die von der Politik bis 2020 geforderte eine Million Elektrofahrzeuge für die deutsche PkwFlotte, worunter sowohl Hybride (d. h. Pkw mit Verbrennungsmotor und unterstützendem Elektromotor) als auch reine Elektrofahrzeuge fallen, führen jedenfalls aus heutiger Sicht zu keinen gravierenden Änderungen in der deutschen Energiewirtschaft. Lediglich bei einer lokalen Häufung von Elektrofahrzeugen - bspw. in einem Straßenzug - könnte im Verteilnetz eine Engpasssituation erreicht werden. Da aber Investitionen in die künftige Kraftwerksstruktur in der Regel die kommenden Dekaden beeinflusst, ist die Berücksichtigung dieser Entwicklung auch im nationalen Kontext unabdingbar.

\subsection{Weitere Herausforderungen}

Neben den oben genannten zu erwartenden Entwicklungen hin zu einem dezentraleren Energiesystem spielen weitere Herausforderungen eine wichtige Rolle, die bei der Ausgestaltung der Energiesysteme und damit auch bei der Energiesystemanalyse zu berücksichtigen sind:

\section{Energieversorgungssicherheit}

Versorgungssicherheit beinhaltet alle Elemente der Wertschöpfungskette: die Bereitstellung von Primärenergieträgern sowie die Umwandlung, Übertragung und Verteilung von Energie. Während eine sichere Umwandlung, Übertragung und Verteilung von Energie abhängig von einem adäquaten Produktionssystem (ausreichend freie Kapazitäten, die die notwendigen technischen Eigenschaften erfüllen) ist, hängt eine sichere Versorgung mit Primärenergie vom Energiemix und damit von der Diversifizierung und der Importabhängigkeit der verschiedenen Primärenergieträgern $\mathrm{ab}$.

Das Wachstum des EU-Primärenergiebedarfs wird im Grünbuch der Europäischen Kommission (2000) von 1998 bis 2030 auf 11 Prozent geschätzt, wobei eine Erhöhung der Energie-Abhängigkeit von 49 Prozent auf 71 Prozent im selben Zeitraum erwartet wird. Vor diesem Hintergrund spielen Primärenergieträger, die zur Versorgungssicherheit beitragen und in der Europäischen Union auch ausreichend vorhanden sind, wie bspw. erneuerbare Energien und heimische Kohle, zukünftig eine wichtige Rolle.

\section{Klimaschutz}

Der Energiesektor trägt zu 27 Prozent der gesamten Treibhausgasemissionen in der EU bei. Aus verschiedenen Gründen ist dieser Sektor von besonderer Bedeutung für die Umsetzung wirksamer Maßnahmen zur Emissionsminderung. Erstens lassen sich Emissionsminderungen innerhalb des Energiesektors relativ günstig im Vergleich zu anderen klimarelevanten Sektoren realisieren. ${ }^{6}$ Darüber hinaus erleichtert die Struktur des Sektors, mit einer relativ kleinen Anzahl von großen Emittenten, die Überwa- 
chung von Emissionen. Klimaschutzbemühungen und Emissionsreduktionspfade sind für die Entwicklung des Energiesystems von wesentlicher Bedeutung. Die quantitativen Methoden der Energiesystemanalyse sind deshalb wichtige Entscheidungshilfen bei der Gestaltung der Emissionshandelssysteme.

\section{Gesellschaftliche Akzeptanz}

Der Erfolg technischer Innovationen hängt in der Regel nicht nur von deren naturwissenschaftlichtechnischer Exzellenz ab, sondern auch davon, dass sich diese im Wettbewerb durchsetzen können und dabei die gesellschaftlichen Kontexte der jeweiligen Herausforderungen adäquat erfasst und eingeschätzt wurden. Dies betrifft z. B. ökonomische Verhältnisse, politische und rechtliche Rahmenbedingungen, ethische Aspekte oder die öffentliche Problemwahrnehmung und Fragen der Technikakzeptanz (s. hierzu auch Grunwald 2005). Gerade der Energiebereich bietet vielfältiges Anschauungsmaterial für die Relevanz derartiger gesellschaftlicher Einflüsse.

Die Energiesystemanalyse und Technikfolgenabschätzung nimmt zum einen technische Entwicklungen und deren Potenziale auf und gibt Rückmeldung zur Weiterentwicklung des Technologieportfolios. Zum anderen bündelt sie technische Fragen auf der Systemebene und betrachtet die Schnittstellen zu gesellschaftlichen Fragen von Energietechnologien und -systemen, um deren Innovationspotenzial beurteilen $\mathrm{zu}$ können (s. auch Grunwald et al. 2008).

\section{Herausforderungen für die Systemanalyse}

Die Energiesystemanalyse soll helfen, Entscheidungen in Energiepolitik und Energieforschung im Hinblick auf Technologien und Infrastrukturen für Energiebereitstellung und Energieumwandlung wissensbasiert und systematisch zu unterstützen. Unter dem Energiesystem kann das globale, das europäische, ein nationales, das eines Stadtteils, eines Industriestandorts oder eines Hauses verstanden sein. Je nach Fragestellung sind unterschiedlichste Faktoren und Rahmenbedingungen zu berücksichtigen. Besonders wichtig erscheint die Erarbeitung in sich konsistenter Energieversorgungsstrategien (meist für Nationen), teilweise auch „Energiezukünfte“ genannt. Dabei ist die Abstimmung der verschiedenen Technologien auf der Angebots- als auch auf der Nachfrageseite von zentraler Bedeutung. Veränderungen an einer Stelle des Energiesystems ziehen zwangsläufig Veränderungen an anderer Stelle nach sich, die bei der Erarbeitung einer in sich konsistenten Energieträgerstrategie berücksichtigt werden müssen. So wird die oben aufgezeigte Dezentralisierung mit einer fluktuierenden Einspeisung einhergehen, insbesondere bedingt durch den Ausbau erneuerbare Energien, welcher zu einem steigenden Bedarf an schnell regelbaren Kraftwerken und/oder Speichern führen wird. Die Energiesystemanalyse kann dabei aufzeigen, welchen Beitrag verschiedene Technologien zum Ausgleich der Schwankungen leisten können und welche ökonomischen Implikationen sich daraus ergeben.

Die Herausforderung ist die adäquate $\mathrm{Ab}$ bildung der verschiedenen Energietechnologien bei Kenntnis ihrer techno-ökonomischen Charakteristika, insbesondere unter Abschätzung des technischen Fortschritts in den nächsten Jahren. ${ }^{7}$ Methodisch müssen hierzu zeitlich und räumlich hoch aufgelöste Modelle eingesetzt werden, die eine Analyse des Energiesystems auf stündlicher Basis erlauben und möglichst einen Betrachtungshorizont von 30 bis 50 Jahren abdecken. Da allerdings über einen solchen Zeitraum eine hohe zeitliche Auflösung in der Regel nicht zielführend ist und in der Regel die entsprechenden Modelle nicht in akzeptabler Rechenzeit gelöst werden können, werden Modelle mit unterschiedlicher zeitlicher Auflösung und unterschiedlichen Planungshorizonten eingesetzt und miteinander gekoppelt. ${ }^{8}$ Zukünftig werden vermutlich aufgrund der Weiterentwicklungen in der Informatik und bei der Hardware integrierte Modelle an Bedeutung gewinnen. Daneben wird die Energiesystemanalyse zukünftig auch die Verteilungsnetze stärker berücksichtigen müssen, da zunehmend auch mit Netzrückwirkungen aufgrund von KWK-Anlagen und Photovoltaik zu rechnen ist. Bisher wurden die Stromnetze, insbesondere die Niederspannungsnetze, in der Energiesystemanalyse nur sehr vereinfacht berücksichtigt. 
Zunehmend wichtiger werden zudem gesellschaftliche Fragen und die gesellschaftliche Diskussion im Zusammenhang mit dem Energiesystem. Der Erfolg technischer Innovationen hängt ganz wesentlich von der gesellschaftlichen Technikakzeptanz $\mathrm{ab}^{9}$. Die Energiesystemanalyse und Technikfolgenabschätzung kann vor diesem Hintergrund Schnittstellen nach zwei Seiten leisten. Zum einem nehmen sie die technischen Entwicklungen und deren Potenziale in ihre Modelle auf und geben Rückmeldung zur Weiterentwicklung des Technologieportfolios. Zum anderen bündeln sie technische Fragen auf der Systemebene und betrachten die Schnittstelle zu gesellschaftlichen Fragen von Energietechnologien und -systemen, um deren Innovationspotenzial beurteilen zu können. Diese Aufgabe ist einerseits erkenntnisorientiert, und führt zu handlungs- und entscheidungsrelevantem Wissen für Gesellschafts-, Politik- und Wirtschaftsberatung, wie bspw. für die Energieund Wettbewerbspolitik. Dabei geht es sowohl um das Wissen über die Folgen von technischen Entwicklungen für die Gesellschaft (z. B. in Bezug auf Chancen und Risiken von Technologien) als auch um den technischen Entwicklungsbedarf, der sich aus gesellschaftlichen Anforderungen (bspw. Forderung einer nachhaltigen Entwicklung) und Problemen (bspw. langfristige Sicherung der Energieversorgung) ergibt. Herausforderung ist hierbei, auch entsprechende Entwicklungen abzuschätzen - wie bspw. der weitere Ausbau erneuerbarer Energien, der maßgeblich durch die politische Förderung (und die gesellschaftliche Akzep$\operatorname{tanz})$ determiniert ist. Energiesystemanalyse und Technikfolgenabschätzung können helfen, Fakten zu einer gesellschaftlichen Diskussion beizusteuern, um damit die Diskussion zu versachlichen. ${ }^{10}$

Die Energiesystemanalyse und Technikfolgenabschätzung leistet somit eine Hilfestellung, um heutige Entscheidungen auf rationaler Basis $\mathrm{zu}$ treffen und um die heutige Vorstellung von der Zukunft strukturiert und wissensbasiert abzubilden. In der Energiesystemanalyse ist der Anwender gezwungen, sich mit den Rahmenbedingungen und Parametern systematisch auseinanderzusetzen und diese transparent und nachvollziehbar darzustellen. Dabei müssen selbstredend die Entwicklungen zu einem dezentraleren Energiesystem berücksichtigt werden. Die
Energiesystemanalyse ist somit eine quantitative Denkhilfe sowie eine Übung zur Informationsgewinnung und hilft damit Entscheidungen auf gut informierter Basis zu treffen.

\section{Anmerkungen}

1) Siehe z. B. Percebois 2009.

2) Zur Einführung in die Energiesystemanalyse wird auf Möst et al. (2008) verwiesen.

3) Die Photovoltaikentwicklung in Deutschland wird in vielen Studien unterschätzt, da insbesondere in den letzten beiden Jahre (2009 und 2010) extreme Zuwächse bei der installierten Kapazität zu verzeichnen waren.

4) Siehe z. B. Dena 2005.

5) Die Lichtblick $A G$ ist ein privat finanziertes deutsches Energieversorgungsunternehmen mit ca. 580.000 Privat- und Gewerbekunden und bezeichnet sich als Marktführer für Ökostrom (s. http://www.lichtblick.de).

6) Vergleich z. B. IPTS 2000 und Mantzos et al. 2003.

7) Siehe hierzu auch Wietschel et al. 2009.

8) Siehe z. B. Rosen et al. 2007 und Möst et al. 2010.

9) Siehe hierzu auch TaTuP-Schwerpunkt „Technikakzeptanz als Gegenstand wissenschaftlicher und politischer Diskussion“" in TATuP 14/3 (2005); http://www.itas.fzk.de/tatup/053/inhalt.htm.

10) Vgl. auch Möst et al. 2008.

\section{Literatur}

BKWK - Bundesverband Kraft-Wärme-Kopplung, 2005: Fakten zur Kraft-Wärme-Kopplung. Berlin

BMU - Bundesministerium für Umwelt, Naturschutz und Reaktorsicherheit, 2009: Erneuerbare Energien in Zahlen - Nationale und internationale Entwicklung

BMU - Bundesministerium für Umwelt, Naturschutz und Reaktorsicherheit, 2007: Das Integrierte Energieund Klimaprogramm der Bundesregierung - IEKP

BMWI - Bundesministerium für Wirtschaft und Technologie, 2010: Energiestatistiken: Endenergieverbrauch nach Anwendungsbereichen; http://www. bmwi.de/BMWi/Navigation/Energie/Statistik-undPrognosen/energiedaten.html (download 5.10.10)

Dena - Deutsche Energie-Agentur, 2005: Energiewirtschaftliche Planung für die Netzintegration von Windenergie in Deutschland an Land und Offshore bis zum Jahr 2020. Endbericht

Europäische Kommission, 2000: Green Paper - Towards a European Strategy For the Security of Energy supply - COM(2000), 29.11.2000. Brüssel 
Europäische Kommission, 2004a: Analysis of Crossborder Congestion Management Methods for the EU Internal Electricity Market. European Commission Directorate-General Energy and Transport. Brüssel

Europäische Kommission, 2004b: Richtlinie 2004/8/ EG des Europäischen Parlaments und des Rates über die Förderung einer am Nutzwärmebedarf orientierten Kraft-Wärme-Kopplung im Energiebinnenmarkt und zur Änderung der Richtlinie 92/42/EWG. Brüssel

Europäische Kommission, 2006: Richtlinie 2006/32/ EG des Europäischen Parlaments und des Rates vom 5. April 2006 über Endenergieeffizienz und Energiedienstleistungen und zur Aufhebung der Richtlinie 93/76/EWG des Rates, Brüssel

Genoese, F.; Klobasa, M.; Wietschel, M., 2009: Zukünftige Entwicklung von erneuerbaren Energien in Deutschland und Anforderungen an das Energiesystem. In: Umweltwirtschaftsforum 4 (2009), S. 307-312

Grunwald, A., 2005: Zur Rolle von Akzeptanz und Akzeptabilität von Technik bei der Bewältigung von Technikkonflikten. In: Technikfolgenabschätzung Theorie und Praxis 14/3 (2005), S. 54-60

Grunwald, A.; Möst, D.; Fichtner, W., 2008: Energiesystemanalyse im KIT Zentrum Energie. In: Möst, D.; Fichtner, W.; Grunwald, A.: Energiesystemanalyse. Karlsruhe

Haubrich, H.-J.; Fritz, W.; Zimmer, C. et al., 2002: Grenzüberschreitende Übertragungskapazitäten und Engpässe im europäischen Stromnetz. In: Energiewirtschaftliche Tagesfragen 52/4 (2002), S. 232-237

IPTS - Institute for Prospective Technological Studies, 2000: Preliminary Analysis of the Implementation of an EU-wide Permit Trading Scheme on CO2 Emissions Abatement Costs: Results from the POLES model, Paper. Sevilla

Mantzos, L.; Capros, P.; Kouvaritakis, N.; ZekaPaschou, M., 2003: European Energy and Transport Trends to 2030. Report produced for DG Transport and Energy. Athen

Möst, D.; Fichtner, W., 2008: Einführung zur Energiesystemanalyse. In: Möst, D.; Fichtner, W.; Grunwald, A. (Hg.): Energiesystemanalyse. Karlsruhe, S. 11-31

Möst, D.; Fichtner, W., 2010: Renewable Energy Sources in European Energy Supply and Interactions with Emission Trading, In: Energy Policy 38/6 (2010), S. 2898-2910

Möst, D.; Fichtner, W.; Grunwald, A. (Hg.), 2008: Energiesystemanalyse. Karlsruhe; http:/digbib. ubka.uni-karlsruhe.de/volltexte/1000011891 (download 6.12.10)
Percebois, J., 2009: Französische Energiepolitik: Von der Unabhängigkeit zur Interdependenz. In: Energiewirtschaftliche Tagesfragen 5 (2009), S. 62-67

Roon, S. von; Steck, M., 2009: Dezentrale Bereitstellung von Strom und Wärme mit Mikro-KWK-Anlagen - Effizienzvorteile, Techniken, Potenziale und das Konzept des virtuellen Kraftwerks. In: uwf - Umweltwirtschaftsforum 17/4 (2009), S. 313-319

Rosen, J.; Tietze-Stockinger, I.; Rentz, O., 2007: Model-based Analysis of Effects from Large-scale Wind Power Production. In: Energy 32/4 (2007). ECOS 05. 18th International Conference on Efficiency, Cost, Optimization, Simulation, and Environmental Impact of Energy Systems - ECOS 05, April 2007, S. 575583, DOI: $10.1016 /$ j.energy.2006.06.022

Wietschel, M.; Dötsch, C.; Herkel, S. et al. (Hg.), 2010: Energietechnologien 2050 - Schwerpunkte für Forschung und Entwicklung. Karlsruhe (ISI-Schriftenreihe Innovationspotenziale)

\section{Kontakt}

Prof. Dr. Dominik Möst

TU Dresden

Lehrstuhl für Energiewirtschaft

Münchner Platz 3, 01062 Dresden

Internet: http://www.ee2.biz 